\title{
Possible supplemental breast cancer screening modalities
}

\author{
Takayoshi Uematsu ${ }^{1}$
}

Published online: 7 September 2016

(c) The Japanese Breast Cancer Society 2016

\section{Overview}

Mammography is the only screening modality that has been proven to reduce breast cancer mortality, apart from access to therapy; however, it is not the perfect screening modality for women with dense breasts. Although digital mammography has been shown to be more sensitive than film-screen mammography for women with dense breasts, there is a scope for improvement. Supplemental breast cancer screening modalities have been proposed to increase the sensitivity and detection rates of early-stage breast cancer in women with dense breasts. However, there are no global guidelines that recommend the use of supplemental breast cancer screening modalities in such women. Therefore, we need to know the current status and clinical pathways regarding possible supplemental breast cancer screening modalities for women with dense breasts.

An appropriate population-based breast cancer screening program based on the balance between cost and benefit should be a high priority. Further research based on evidence-based medicine for possible supplemental breast cancer screening modalities is encouraged. Furthermore, it is very important that the ethnicity, workforce, workflow, and resources for breast cancer screening in each country should be taken into account when considering supplemental breast cancer screening modalities for women with dense breasts. Based on the current situation of breast imaging in Japan, the possible supplemental breast cancer

Takayoshi Uematsu

t.uematsu@scchr.jp

1 Breast Imaging and Breast Intervention Section, Shizuoka Cancer Center Hospital, Naga-izumi, Shizuoka 411-8777, Japan screening modalities are ultrasonography, digital breast tomosynthesis, and breast magnetic resonance imaging (MRI).

The special articles in this issue of Breast Cancer are devoted to supplemental breast cancer screening modalities, especially for women with dense breasts. These special articles represent the collective efforts of many individuals and I wish to thank all of the contributors.

I reviewed the current status and clinical pathways regarding possible supplemental breast cancer screening modalities for women with dense breasts from the point of view of population-based breast cancer screening programs in Japan [1].

Professor Skaane $\mathrm{P}$ contributed the article entitled "Breast cancer screening with digital breast tomosynthesis" [2].

They will serve as excellent reference sources for anyone interested in possible supplemental breast cancer screening modalities.

\section{References}

1. Uematsu T. The need for supplemental breast cancer screening modalities: a perspective of population-based breast cancer screening programs in Japan. Breast Cancer. 2016. doi:10.1007/ s12282-016-0707-2 (Epub ahead of print).

2. Skaane P. Breast cancer screening with digital breast tomosynthesis. Breast Cancer. 2016. doi:10.1007/s12282-016-0699-y (Epub ahead of print). 